\section{Jahresbericht der SMIFK 1999}

\author{
A. Campana ${ }^{a}$, HP. Kuhn ${ }^{b}$ \\ a SMIFK-Präsident, Genf \\ b SMIFK-Sekretär, Bern
}

Die Schweizerische Medizinische Interfakultätskommission behandelte im Berichtsjahr an drei Sitzungen folgende Themen:

\section{Akkreditierung der Fakultäten}

In der unhelvetischen Frist von zwei Jahren wurde das Pilotprojekt der Akkreditierung von Fakultäten an allen fünf medizinischen Fakultäten der Schweiz durchgezogen. Die resultierende Analyse der Stärken und Schwächen der Infrastruktur und Prozessqualität der medizinischen Ausbildung wurde von den Fakultäten als wichtig und positiv erlebt: Bereits die interne Erstellung des Berichtes zuhanden der Experten und die nachfolgende in ihrer Qualität überzeugende Arbeit der Experten ergaben eine wertvolle Bilanz der aktuellen Situation und des Handlungsbedarfs.

\section{Studienreform}

Die unter der Leitung von Prof. Wauters, Lausanne, arbeitende Kommission Studienreform legte in der September-Sitzung im Sinne eines Zwischenberichts ein Konzept zur Harmonisierung der Studienprogramme und der Evaluation vor. Die SMIFK-Delegierten nahmen grossmehrheitlich positiv Stellung zum vorgeschlagenen Kurs.

Das zweite Konzept enthielt Vorschläge zum Thema Beschränkung des Studienzugangs. Trotz längerer Grundsatzdiskussionen konnte hier im Rahmen der

Korrespondenz:

HP. Kuhn

Fürsprecher

SMIFK

CH-3000 Bern 16
SMIFK (noch) kein Konsens über die Alternativen bzw. Kombination der Eignungsprüfung vor Studienantritt bzw. nach den ersten Semestern gefunden werden.

\section{Studienplatzkapazität an den medizinischen Fakultäten der Schweiz}

Ins Berichtsjahr fielen die entscheidenden Aktivitäten in diesem Bereich: Es geht darum, ein schweizerisch einheitliches Planungsinstrument zuhanden der Fakultäten zu schaffen, das diesen erlaubt, die für die Ausbildung notwendigen Ressourcen in Abhängigkeit von Studentenzahlen und gewünschten bzw. geforderten Unterrichtsformen zu belegen. Die Ergebnisse sollen im März 2000 vorliegen.

\section{Koordination des Wahlstudienjahres}

1998 hatte die SMIFK festgestellt, dass die Vergabe der Wahlstudienjahrplätze in der Romandie gut funktioniert, während in der deutschsprachigen Schweiz die Koordination zwischen den Fakultäten und den Spitälern, in denen das Wahlstudienjahr absolviert wird, noch nicht in erwünschtem Masse funktioniert. Im Berichtsjahr 1999 sind hier nun die entscheidenden Koordinationsarbeiten in den deutschschweizer Fakultäten in Angriff genommen worden.

\section{Armeereform und Koordination mit dem Medizinstudium}

Die SMIFK-Delegierten haben sich im November vom Oberfeldarzt über die Reform im Bereich der Sanitätstruppen unterrichten lassen. Im Hinblick auf eine optimale Koordination zwischen Armeereform und Studienplanung wurde eine Kommission mit Vertretern der SMIFK, der Armee, des VSA0 und der FMH eingesetzt. Ziele sind die zeitliche Koordination der Ausbildungsschulen mit Semesterbeginn und Ende an den Fakultäten sowie Fragen der gegenseitigen Anrechnung von Ausbildungen.

\section{Verpflichtung der Fakultäten zur Weiterbildung}

Verschiedene revidierte Universitätsgesetze verpflichten die Fakultäten zur Weiterbildung. Unklar ist dabei in der Regel unter anderem die Finanzierungsfrage. Die SMIFK hat an ihrer November-Sitzung auch zu diesem Thema eine Arbeitsgruppe mit Vertretern der Fakultäten, der FMH, des VSAO und des VSM eingesetzt (aber im März wieder aufgelöst zugunsten einer verstärkten Mitsprache von Fakultätsdelegierten in der KWFB). 


\section{Klage von Genfer Studenten betr. MC-Prüfung; Vorarbeiten für schweizerischen Lernzielkatalog}

Auch die SMIFK wurde über die entsprechende Klage von Genfer Studenten orientiert. Sie nimmt diese Probleme sehr ernst, konnte aber feststellen, dass der leitende Ausschuss die in seine Zuständigkeit fallenden Massnahmen in die Wege geleitet hat. Hingegen hat die SMIFK in diesem Zusammenhang erkannt, wie dringend notwendig ein gesamtschweizerischer Lernzielkatalog für das Studium ist. Sie hat den entsprechenden Auftrag für Vorarbeiten an die SMIFKKommission Studienreform delegiert.

\section{Prüfungsgebühren für das 13. Semester}

Die SMIFK hat festgestellt, dass Medizinstudenten und Medizinstudentinnen teilweise für das 13. Semester Immatrikulationsgebühren bezahlen mussten. Sie hat an ihrer Novembersitzung die Schweizerische Hochschulkonferenz gebeten, die notwendigen Massnahmen zu ergreifen (das Medizinstudium dauert reglementsgemäss 6 und nicht $6 \frac{1}{2}$ Jahre; im 13. Semester werden keine Lehrveranstaltungen angeboten, sondern ausschliesslich Prüfungen abgelegt, für die Prüfungsgebühren erhoben werden. Es scheint der SMIFK deshalb weder ökonomisch noch rechtlich gerechtfertigt, nach dem 12. Semester Immatrikulationsgebühren zu erheben).

\section{Fakultäre Weiterbildungstitel}

Die SMIFK hat an ihrer November-Sitzung zustimmend von einer Aussprache zwischen den Dekanen und der FMH zu dieser Frage Kenntnis genommen. Fakultäre Weiterbildungstitel können primär ein Bedürfnis sein für ausländische Ärzte ausserhalb des EU-/EWR-Raumes. Sie sollen in Anwendung der FMH- und zukünftig Bundesweiterbildungsordnung und nach Prüfung der Unterlagen durch die FMHTitelkommission von den Fakultäten erteilt werden. Damit ist gewährleistet, dass dieselben Kriterien wie für schweizerische Weiterbildungstitel angewendet werden.

\section{Vernehmlassungen}

In der März-Sitzung hat die SMIFK zur aktuellen KVGTeilrevision Stellung genommen und ihrer Sorge darüber Ausdruck gegeben, ob mit der vorgeschlagenen Revision die Aus- und Weiterbildung der Ärzte auch in Zukunft finanziell sichergestellt werden kann.
Ebenfalls in der März-Sitzung hat sie zur Revision des Freizügigkeitsgesetzes Stellung genommen (als Gast Prof. Dr. Thomas Fleiner): Die Revision des FG macht das Medizinalberufegesetz nicht überflüssig; es sollte im Gesetz ausdrücklich festgeschrieben werden, bis wann der Bundesrat das Medizinalberufegesetz dem Parlament vorlegen muss. Sprachprüfungen sind notwendig, weil die multikulturelle Schweiz ein Bedürfnis nach Ärzten hat, die der Landessprachen mächtig sind. Die Koordination der Weiterbildung durch eine Trägerorganisation wird begrüsst - die FMH sollte allerdings diese Aufgabe in Zusammenarbeit mit der SMIFK erfüllen. Angesichts einer nicht unwahrscheinlichen erheblichen Ärztezuwanderung aus dem Ausland sollte während der zweijährigen Übergangsfrist und während der drei nachfolgenden Jahre mit Vorzugskontingenten für EU-Angehörige im Interesse der Finanzierbarkeit der Sozialversicherung eine restriktive Bewilligungspraxis verfolgt werden.

Im September hat die SMIFK zum Vorentwurf des Bundesgesetzes über die universitäre Ausbildung in den medizinischen Berufen (MedBG-Ausbildung) Stellung genommen. Die allgemeine Stossrichtung wurde begrüsst. Das Kernstudium sollte nicht höchstens, sondern mindestens $80 \%$ umfassen. Das höchstens 20\% umfassende Mantelstudium sollte weitgehend in den letzten Studienjahren stattfinden, damit die Studierenden mit ihren sehr unterschiedlichen Ausgangsbasen in den ersten Studienjahren auf ein homogenes Niveau gebracht werden können. Angesichts der spezifischen Bedürfnisse im Bereich der Medizin muss es eine eigene Akkreditierungsinstanz geben.

Gleichwertigkeit von ausländischen Diplomen aus nicht EU-/EWR-Staaten: Zu dieser Frage hat das BAG eine Stellungnahme der SMIFK gewünscht. Die SMIFK stellt eingangs fest, dass es um seltene Fälle geht, kommt doch eine Gleichwertigkeitsbescheinigung eines nicht EU-/EWR-Diplomes nur dann in Frage, wenn der Kanton eine Unterversorgung (Bedürfnisklausel) bereits festgestellt hat. Ab allfälligem Inkrafttreten der bilateralen Abkommen ist nicht mehr zu erwarten, dass es überhaupt noch Anwendungsfälle geben wird. Die SMIFK spricht sich grundsätzlich für die vorgeschlagene pragmatische Lösung aus, allerdings mit dem Zusatz, es sei bei der Gleichwertigkeitsbescheinigung jeweils die Auflage zu machen, innerhalb von fünf Jahren das abgekürzte Staatsexamen nachzuholen, was voraussetzt, dass diese Ärzte und Ärztinnen künftig zum Staatsexamen zugelassen werden. 